\title{
Relationship between cognitive impairment and serum amyloid $\beta$-protein, adiponectin, and C-reactive protein levels in type II diabetes patients
}

\author{
Haiju Liu ${ }^{1 \#}$, Ji Ma ${ }^{2 \#}$, Li Sun ${ }^{3}$, Qin $\mathrm{Zhang}^{4}$, Jiubo Fan $^{4}$ \\ ${ }^{1}$ Department of Outpatient Office, Xiangyang Central Hospital , Affiliated Hospital of Hubei University of Arts and Science, Xiangyang, China; \\ ${ }^{2}$ Department of Endocrinology, Xiangyang Central Hospital, Affiliated Hospital of Hubei University of Arts and Science, Xiangyang, China; \\ ${ }^{3}$ Medicine of Xiangyang Vocational and Technical College, Xiangyang, China; ${ }^{4}$ Department of Clinical Laboratory, Xiangyang Central Hospital, \\ Affiliated Hospital of Hubei University of Arts and Science, Xiangyang, China \\ Contributions: (I) Conception and design: H Liu; (II) Administrative support: J Fan; (III) Provision of study materials or patients: J Ma; (IV) Collection \\ and assembly of data: H Liu, L Sun; (V) Data analysis and interpretation: J Ma, Q Zhang; (VI) Manuscript writing: All authors; (VII) Final approval \\ of manuscript: All authors. \\ "These authors contributed equally to this work. \\ Correspondence to: Jiubo Fan. Department of Clinical Laboratory, Xiangyang Central Hospital, Affiliated Hospital of Hubei University of Arts and \\ Science, Xiangyang 441021, China. Email: fanjiubo@126.com.
}

Background: This study sought to explore the plausible mechanism of the cognitive impairment of patients with type 2 diabetes by analyzing the levels of serum amyloid $\beta$-protein (A $\beta 1-42)$, adiponectin, and C-reactive protein (CRP).

Methods: Eighty-four patients diagnosed with type 2 diabetes and 60 healthy people were selected as the participants for this study. Clinical data were collected using self-made questionnaires. The Repeatable Battery for the Assessment of Neuropsychological Status (RBANS) scale was used to access cognitive functions. The serum A $\beta 1-42$ and adiponectin levels were individually determined by an enzyme-linked immunoassay (ELISA). CRP was detected using a Siemens BNP II specific-protein analyzer.

Results: Patients in the case group had significantly lower immediate memory, visual span, speech function, attention, and delayed memory scores on the RBANS scale than patients in the control group. The A $\beta 1-42$ levels of patients in case group were significantly higher than those of patients in the control group. The age of patients was significantly negatively correlated to RBANS scale subtest scores and standard scores in the case group. The number of years of schooling was significantly positively correlated to immediate memory, visual span, attention, delayed memory scores, and standard scores, but negatively correlated with speech function. After adjusting for age and the number of years of schooling, the A $\beta 1-42$ levels of patients in the case group were significantly negatively correlated with immediate memory, attention, delayed memory scores, and standard scores $(\mathrm{P}<0.05)$. The adiponectin levels of patients in the case group were positively correlated with RBANS scale subtest scores and standard scores $(\mathrm{P}<0.05)$.

Conclusions: Type 2 diabetes patients suffered from cognitive impairment. It appears that the mechanism may be associated with increased serum A $1-42$ levels, decreased adiponectin levels and inflammation reaction. The detection of serum A $\beta 1-42$ and adiponectin could be used as indicators of the degree of cognitive impairment in patients with type 2 diabetes.

Keywords: Amyloid $\beta$-protein-42 (Aß1-42); diabetes; cognition; function; damage

Submitted Apr 01, 2021. Accepted for publication Jun 11, 2021.

doi: 10.21037/apm-21-1074

View this article at: https://dx.doi.org/10.21037/apm-21-1074 


\section{Introduction}

With an estimated 400 million people affected by diabetes worldwide, diabetes mellitus (DM) is a global epidemic (1). Further, with the increase in people's living standards and living pressure, diabetes has become a major threat to young people. There are two major types of DM, including insulin-dependent diabetes [type 1 DM (T1DM)] and insulin-independent diabetes [type 2 DM (T2DM)]. Approximately, 90\% of people with diabetes have T2DM. The main damage of diabetes includes long-term poor glycemic control, which leads to complications, including kidney disease, hypertension, infection, and diabetic foot (2). Further, diabetes is often associated with cognitive impairment, decreased learning and memory ability, reduced understanding and judging ability, and even dementia (3). The risk of dementia for T2DM diabetes patients is about 1.5 to 2.0 times higher than that in the normal population. DM possesses a bidirectional relationship with major depressive disorder (MDD) (4). MDD has significant effects on the course and outcome of diabetes (5). Some studies have shown that MDD has a specific association with T2DM (6). Similarly, elevated depressive symptoms have been associated with a higher risk of developing T2DM (7). However, the connection between these two conditions is poorly characterized. Diabetes is closely associated with cognitive impairment, and the mechanism is complicated. A series of pathological changes resulting from glycol metabolic disorder or insulin resistance can cause hippocampus, other memory, study-related tissue, and organ damage, which in turn can cause cognitive impairment. In previous study, it was reported that serum levels of adiponectin and 8-OHdG had a close relation to the neurological cognitive impairment of the elderly T2DM patients. However, the study was limited to two specific factors. In our study, we investigated the comprehensive mechanisms of cognitive impairment in T2DM from various views. Amyloid beta protein $(A \beta)$ is the main component of amyloid plaques in the brains of people with Alzheimer's disease (AD), which can kill brain cells. It has been reported that it plays an important role in cognitive impairments in different kinds of brain diseases. This study provided evidence-based medicine for related mechanism and early cognitive impairment screening by analyzing the connection between T2DM patients' cognition and serum amyloid $\beta$-protein-42 (A $\beta 1-42$ ) levels. We present the following article in accordance with the STROBE reporting checklist (available at http://dx.doi.org/10.21037/apm-211074).

\section{Methods}

\section{Sample source}

Eighty-four patients (comprising 50 males and 34 females, aged from 25 to 75) diagnosed with T2DM at the Department of Endocrinology, Xiangyang Central Hospital from January to December 2014 were selected for the case group. The selected patients were tested twice for fasting blood glucose (GLU), which conforms with the diagnostic criteria for T2DM set out in the 2013 China guidelines. Healthy patients (comprising 40 males and 20 females, aged from 28 to 72 ) from a medical examination center were chosen as members of the control group. Patients in the control group had no history of diabetes and no family history of diabetes, and their blood GLU levels were between 3.9 and $6.1 \mathrm{mmol} / \mathrm{L}$. The study was conducted in accordance with the Declaration of Helsinki (as revised in 2013). Informed consent was obtained from all individual participants, and ethical approval was waived by the Xiangyang Central Hospital, Affiliated Hospital of Hubei University of Arts and Science.

\section{Exclusion criteria for the two groups}

Patients were excluded from the study if they met any of the following exclusion criteria: (I) had an association with an acute complication of diabetes; (II) had congenital mental retardation, multiple occurrences of cerebral stroke, or epilepsy or a diagnosis of insanity; (III) were taking antidepression drugs or immuno-suppressants; (IV) had severe liver and kidney dysfunction; (V) had 2 years of alcohol dependence or a drug-abuse dependence; (VI) had a history of malignancy or infection; (VII) and/or refused to be in the group or did not fit the scale testers.

\section{Clinical data collection}

The data of patients in the two groups were collected, including data in relation to gender, age, years of education, body mass index (BMI), recording fasting GLU, total cholesterol (TC), low-density lipoprotein cholesterol (LDL-C), and glycosylated hemoglobin $\left(\mathrm{HbA}_{1 \mathrm{C}}\right)$.

\section{The Repeatable Battery for the Assessment of Neuropsychological Status (RBANS) scale}

A trained physician used the RBANS scale to evaluate the cognitive function of the patients. The patients completed 
Table 1 Comparison of clinical data $(\bar{x} \pm s)$

\begin{tabular}{lcccccccc}
\hline Group & No. & Age & Years of schooling & TC $(\mathrm{mmol} / \mathrm{L})$ & $\mathrm{LDL}-\mathrm{C}(\mathrm{mmol} / \mathrm{L})$ & $\mathrm{BMI}\left(\mathrm{kg} / \mathrm{m}^{2}\right)$ & $\mathrm{GLU}\left(\mathrm{mmol}^{\prime} \mathrm{L}\right)$ & $\mathrm{HbA} \mathrm{AC}_{1 \mathrm{C}}(\%)$ \\
\hline Case group & 49 & $52.0 \pm 13.2$ & $11.2 \pm 5.3$ & $4.8 \pm 0.9$ & $3.5 \pm 0.8$ & $25.4 \pm 2.6$ & $5.1 \pm 0.9$ & $43.8 \pm 12.1$ \\
Control group & 42 & $51.1 \pm 11.8$ & $10.7 \pm 4.7$ & $4.7 \pm 1.1$ & $3.4 \pm 1.0$ & $22.6 \pm 4.1$ & $8.2 \pm 2.2$ & $41.7 \pm 10.6$ \\
T value & & 0.41 & 0.27 & 0.48 & 0.53 & 4.67 & 8.48 \\
P value & & 0.81 & 0.72 & 0.55 & 0.65 & 0.02 & 0.01 & 0.01 \\
\hline
\end{tabular}

TC, total cholesterol; LDL-C, low-density lipoprotein cholesterol; BMI, body mass index; GLU, glucose; $\mathrm{HbA}_{1 \mathrm{C}}$, glycosylated hemoglobin.

the subtest in 30-40 min, including questions on immediate memory (memory studying and story retelling), visual span (graphic copying and line positioning), speech functions (pattern naming and semantic fluency), attention (digit span, and test coding), and delayed memory (vocabulary memory, vocabulary recognition, story recall, and graphic recall). The scale scores were converted based on the different ages of the patients, and then converted to standard scores.

\section{Self-Rating Depression Scale (SDS)}

A trained physician administered the Self-Rating Anxiety Scale (SAS) and SDS tests to patients. The SDS and SAS were established by Zung in 1971. A total of 20 items and a 4-point score were used (in which forward scoring and reverse scoring were used for one-half each). The questionnaires were simple and used to evaluate the depression and degree of anxiety of the patients. $\geq 50 \%$ of patients had depression or anxiety, and $<50 \%$ of patients did not have depression or anxiety.

\section{Serum Aßl-42, adiponectin, and C-reactive protein (CRP) level assays}

Five $\mathrm{mL}$ of fasting blood from each patient in the two groups was collected and centrifuged for $10 \mathrm{~min}$. The serum was collected and stored at $-80{ }^{\circ} \mathrm{C}$. The serum A $\beta 1-$ 42 and adiponectin levels were individually determined by an enzyme-linked immunoassay (ELISA) (Jingmei Biocompany). CRP was detected by Siemens BNP II specific protein analyzer.

\section{Statistical Analysis}

SPSS 20.0 software was used for the statistical analysis. The normal distributed measurement data were expressed as $(\bar{x} \pm$ s). $T$-tests were conducted to compare the two groups. Nonnormal distributed measurement data were expressed as $M$
(P25, P75). Non-parametric tests were used to compare the two groups. A count-data analysis used an $\chi^{2}$ test. A $\mathrm{P}<0.05$ was considered statistically significant.

\section{Results}

\section{Comparison of clinical data in the two groups}

Gender, age, the number of years of education, and the TC and LDL-C levels of the two groups were compared; however, no significant differences were found. The levels of BMI, GLU, and $\mathrm{HbA}_{1 \mathrm{C}}$ in the case group were significantly higher than those in the control group $(\mathrm{P}<0.05)$ (see Table 1).

\section{Comparison of the RBANS scale scores and serum Aßl-42 levels in the two groups}

The immediate memory, visual span, speech function, attention, and delayed memory scores on the RBANS scale and the standard scores of patients in the case group were lower than those of patients in the control group; the difference was statistically significant $(\mathrm{P}<0.05)$ (see Table 1 ). The A $1-42$ levels of patients in the case group were higher than those of patients in the control group; the difference was statistically significant $(\mathrm{P}<0.05)$ (see Table 2$)$.

\section{Correlation efficient between age, number of years of education, serum ABl-42, adiponectin, and CRP levels of the case group and RBANS scale scoring}

The ages of patients in the case group and the RBANS scale subtest scores and standard scores were negatively correlated $(\mathrm{P}<0.05)$. The number of years of schooling was positively related with immediate memory, visual span, attention, and delayed memory scores and standard scores, but negatively correlated with speech function $(\mathrm{P}<0.05)$. After adjusting for age and the number of years 


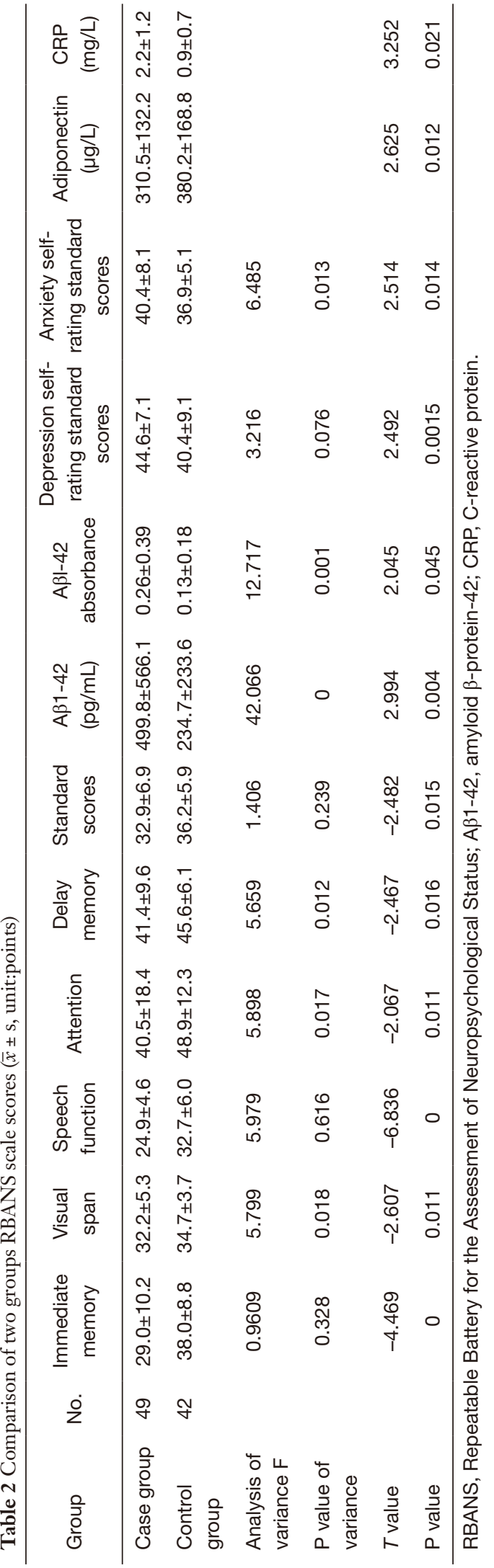

of schooling, the A $\beta 1-42$ levels of patients in the case group were found to be negatively correlated with immediate memory, attention, delayed memory scores, and standard scores $(\mathrm{P}<0.05)$. The adiponectin levels of patients in the case group were positively correlated with the RBANS scale subtest scores and standard scores $(\mathrm{P}<0.05)$ (see Table 3).

\section{Discussion}

Cognition refers to the human brain, which accepts information from the outside world, and processes and converses with internal psychological activities to obtain knowledge or apply knowledge processes, including those related to memory, language, visual space, implementation, calculation, understanding, and judging. Cognitive impairment (also known as cognitive decrease, cognitive deficits, or cognitive disability) refers to damage to the above-mentioned cognitive functions that does not affect an individual's social occupation or daily life, and cannot be explained by known medical or psychiatric disorders. It is a clinical state between normal aging and mild dementia. When two or more are involved in the above cognitive domain, which affect the daily or social capacities of individuals, this can be diagnosed as dementia. Cognitive impairment is a type of mental disorder disease, a cognitive deficit or a disorder.

Diabetes-related cognitive impairment is characterized by acquired cognitive and behavior deficits. T1DM and T2DM can cause cognitive deficits. T1DM mainly manifests as study memory, solving problems and psychomotor speed decreased slightly. In addition to a decline in studying and memory capacity, T2DM cognitive impairment also manifests as a moderate decline in the degree of language memory or complex information processing capacity; for example, an individual's attention, performing ability, spatial processing capacity, and memory may decrease but with few on motor reaction time and immediate memory affected. T2DM has been considered an independent risk factor of mild cognitive impairment, and the occurrence rate of T2DM patients with mild cognitive impairment is $10.8-17.5 \%$. Mild cognitive impairment can progress to Alzheimer and vascular dementia. Studies have shown that patients at risk of T2DM are more than twice as likely to progress from mild cognitive impairment to Alzheimer disease than non-diabetic patients. Cognitive impairment can be assessed and quantified by a specific cognitive function screening scale. The RBANS scale is a tool developed by Randolph to screen for Alzheimer's 
Table 3 Correlation efficient between age, the number of years of education, serum A $\beta 1-42$, Adiponectin, and CRP levels of case group and RBANS scale scoring (r)

\begin{tabular}{|c|c|c|c|c|c|c|}
\hline Variables & Immediate memory & Visual span & Speech function & Attention & Delayed memory & Standard scores \\
\hline Years of schooling & 0.665 & 0.352 & -0.456 & 0.446 & 0.406 & 0.688 \\
\hline$A \beta \mid-42^{*}$ & -0.521 & -0.122 & -0.212 & -0.255 & -0.388 & -0.692 \\
\hline Adiponectin & 0.582 & 0.477 & 0.125 & 0.378 & 0.495 & 0.357 \\
\hline
\end{tabular}

* indicates that age and years of education were adjusted in the row. A $\beta 1-42$, amyloid $\beta$-protein-42; CRP, C-reactive protein; RBANS,

Repeatable Battery for the Assessment of Neuropsychological Status.

disease and has good recognition effects of cognitive impairment caused by various reasons.

Patients were scored in groups by a physician from the Endocrinology Department. Patients from the control group were selected from the medical examination center by the same physician. $\beta$-amyloid precursor protein is hydrolyzed by $\alpha-, \beta-$, and $\gamma$-secretase enzyme to two major metabolites (i.e., $\mathrm{A} \beta 0-40$ and $\mathrm{A} \beta \mathrm{l}-42$ ). In a normal situation, $\mathrm{A} \beta 0-40$ is the main in the brain. $A \beta 1-42$ is generated by amyloid precursor protein hydrolysis in the presence of the $\beta$-secretase enzyme and can be produced by tissue cells from the whole body. Excess soluble monomeric A $\beta 1-42$ gradually aggregates to form a toxic fibrosis precipitate, which induces and participates in a series of pathological changes.

The results of this study showed that the RBANS scale subtest scores and standard scores of patients in the case group were lower than those of patients in the control group, which suggested that the T2DM patients had cognitive impairment. Table 3 shows that serum A $\beta 1-42$ levels and RBANS scale standard scores were negatively correlated, which suggests that an elevated A $\beta 1-42$ level is associated with cognitive impairment. After adjusting for age and the number of years of education, a partial correlation analysis showed a correlation between A $\beta 1$ 42 levels and RBANS scale standard scores. There was a positive correlation between the number of years of education and immediate memory, visual span, attention, delayed memory scores, and standard scores, indicating that patients with a high level of education and strong learning ability could improve themselves by adapting to their environments. Changes in A $\beta 1-42$ levels in T2DM patients influence their cognitive function.

Based on these results, we speculate that under this possible mechanism: (I) A $\beta$ and insulin compete for insulindegrading enzyme (IDE), which is the key enzyme in the degradation process of $A \beta$. Conversely, $A \beta$ and insulin compete for IDE, and the prior option of IDE is insulin. Thus, $\mathrm{A} \beta$ degradation decreases when insulin increases (8), which results in a relative increase of A $\beta 1-42$. The gathering of excess A $1-42$ further exacerbates insulin resistance and oxidative stress, resulting in a vicious cycle of $A \beta 1-42$ production and accumulation, which leads to senile plaque and neurodegenerative disease; (II) disorder transportation across the blood brain barrier of $A \beta 1-42$ leads to $A \beta 1$ 42 deposition in the brain tissues, and white matter and hippocampus formation damage, which results in central conduction system damage. The hypoglycemic state interferes with the brain's energy metabolism, resulting in selective neuronal necrosis and hippocampal atrophy; recurrent hypoglycemia may cause cumulative damage to the brain. That is the main reason for persistent cognitive impairment (9); (III) a variety of inflammatory signaling pathway abnormalities result in the imbalanced expression of inflammatory factors (TNF $\alpha$, IL-1, and IL-6), and the over-activation of the endogenous inflammatory response in the nervous system, which also leads to various degenerative brain pathology changes, such as neuronal apoptosis, $A \beta$ deposition, and neuro-fibrillar tangles. This is one of the start factors of the diabetes cognitive impairment progress $(10,11)$. TNF $\alpha$ is a proinflammatory cytokine with multiple performance. It is produced by activated microglia. The release of cytotoxicity substances and other inflammatory cytokines causes elevated general levels of inflammatory cytokines and induces more glial cells to produce free radical, which causes damage to more neuron and death of a large number of glial cells. Less $A \beta$ phagocytosis results in $A \beta$ deposition in the brain. The excessive accumulation of A $\beta 1-42$ in the brain forms oligomers and adheres to neurons, specifically acting on the synapses and disturbing signal transmission between neurons, causing 
significant memory decline. Imaging can show that bilateral hippocampal volume reduction and extensive brain atrophy exist in T2DM patients and associated with the cognitive decline degree.

Adipose tissue is an active endocrine organ. Adiponectin is one of the adipokines from adipose tissue that exerts biological effects mainly by combining with its acceptors Adipo R1 and Adipo R2. These two acceptors are expressed in skeletal muscle, liver, pituitary, and brain endothelial cells (12). Adiponectin is associated with variety of diseases, including diabetes, obesity, abnormal lipid metabolism, hypertension, and other cardiovascular and neurodegenerative diseases. Adiponectin functions as an endocrine hormone, and can improve insulin sensitivity, insulin resistance, anti-intimal hyperplasia, lipid metabolism, anti-hyperglycemia, anti-inflammatory, and anti-atherosclerosis. Insulin is a neurotrophic factor. A long-term serious shortage of insulin can cause neuron degeneration. Schrijvers et al. found that daily intranasal insulin therapy can improve mild cognitive impairment of which forgetfulness is the main symptom (13). These results showed that memory function changes with $A \beta 1-42$ levels, and the altered ratio of the tau protein and $A \beta 1-42$.

Table 2 shows that the serum adiponectin levels of T2DM patients in the case group were significantly lower than those of patients in the control group; however, these results should be contrasted to those for A42 levels. Table 3 shows that patients' adiponectin levels were positively correlated with their RBANS scale subtest scores and standard scores (14). Thus, adiponectin and A $\beta 1-42$ appear to be risk factors of cognitive impairment. Adiponectin also affects mood state, anti-depression, enhances memory, improves cognitive function and has other beneficial effects (15). Related studies have shown that adiponectin may improve the blood-brain barrier function and brain energy supply, protect cells, inhibit neuronal apoptosis via the generation and accumulation of A $\mathrm{Bl}-42$, and have other mechanisms that are involved in improving cognitive impairment (16).

CRP is a typical acute phase protein synthesized in the liver. Patients with tissue injury or infection show a sharp rise in CRP levels in the body. CRP is a sensitive marker of inflammation and is involved in a variety of human development of inflammation. Studies have shown that abnormal high CRP expression can be detected in the senile plaques and neuro-fibrillary tangles of Alzheimer patients. The abnormal high expression levels of inflammatory cytokines in elderly patients with T2DM may eventually lead to cognitive dysfunction, as significantly higher levels of inflammatory factors may prompt the tau protein phosphorylation and be associated with fibrous $\mathrm{Ab}$ deposition and undernourished neurite formation $(17,18)$.

In short, T2DM could lead to cognitive impairment, and the process of its occurrence is associated with A $11-42$ serum levels remaining higher for a long time, adiponectin reduction, and elevated CRP levels, which are induced by inflammation factors. Adiponectin can lead to cognitive impairment by A $\beta 1-42$ deposition occurrence and aggravation, resulting in decreases in patients' learning and living abilities, and, in severe cases, it can develop into dementia. Thus, it is recommended that patients newly diagnosed with T2DM be monitored regularly, and necessary interventions be implemented to delay the occurrence of cognitive impairment to improve the quality of life of diabetes patients.

T2DM significantly impairs cognitive function in elderly patients. The effects of DM metabolism on cognitive functions in elderly patients should be taken seriously. Prevention should be used to reduce the prevalence of Alzheimer's disease and delay its progression effectively. In conclusion, T2DM patients suffer from cognitive impairment, and the mechanism may be associated with increased levels of serum A $\beta 1-42$, decreased adiponectin, and an inflammation reaction. The detection of serum A $\beta 1-$ 42 and adiponectin could be used as an indicator of the degree of cognitive impairment in T2DM patients.

\section{Acknowledgments}

Funding: This study was supported by the Health and Family Planning Commission of Hubei Province Project (WJ2015XB019 2015-2016), the Research and Development Project of Xiangyang City [2014], the Key Projects of Xiangyang City's Medical and Health Science and Technology Plan in 2020 (2020YL06), and the Xiangyang Medical and Health Guiding Science and Technology Project in 2019 (2019zd26).

\section{Footnote}

Reporting Checklist: The authors have completed the STROBE reporting checklist. Available at http://dx.doi. org/10.21037/apm-21-1074 
Data Sharing Statement: Available at http://dx.doi. org/10.21037/apm-21-1074

Conflicts of Interest: All authors have completed the ICMJE uniform disclosure form (available at http://dx.doi. org/10.21037/apm-21-1074). The authors have no conflicts of interest to declare.

Ethical Statement: The authors are accountable for all aspects of the work in ensuring that questions related to the accuracy or integrity of any part of the work are appropriately investigated and resolved. The study was conducted in accordance with the Declaration of Helsinki (as revised in 2013). Informed consent was obtained from all individual participants, and ethical approval was waived by the Xiangyang Central Hospital, Affiliated Hospital of Hubei University of Arts and Science.

Open Access Statement: This is an Open Access article distributed in accordance with the Creative Commons Attribution-NonCommercial-NoDerivs 4.0 International License (CC BY-NC-ND 4.0), which permits the noncommercial replication and distribution of the article with the strict proviso that no changes or edits are made and the original work is properly cited (including links to both the formal publication through the relevant DOI and the license). See: https://creativecommons.org/licenses/by-nc-nd/4.0/.

\section{References}

1. Herman WH. The global burden of diabetes: an overview. In: Dagogo-Jack S. editor. Diabetes Mellitus in Developing Countries and Underserved Communities. Cham: Springer, 2017:1-5.

2. Eyes on Diabetes. Word diabetes day. International Diabetes Federation 2016.

3. Carracher AM, Marathe PH, Close KL. International Diabetes Federation 2017. J Diabetes 2018;10:353-6.

4. Pan A, Lucas M, Sun Q, et al. Bidirectional association between depression and type 2 diabetes mellitus in women. Arch Intern Med 2010;170:1884-91.

5. Lustman PJ, Clouse RE. Depression in diabetic patients: the relationship between mood and glycemic control. J Diabetes Complications 2005;19:113-22.

6. Nouwen A, Nefs G, Caramlau I, et al. Prevalence of depression in individuals with impaired glucose metabolism or undiagnosed diabetes: a systematic review and meta-analysis of the European Depression in Diabetes (EDID) Research Consortium. Diabetes Care 2011;34:752-62.

7. Demakakos P, Pierce MB, Hardy R. Depressive symptoms and risk of type 2 diabetes in a national sample of middle-aged and older adults: the English longitudinal study of aging. Diabetes Care 2010;33:792-7.

8. Koekkoek PS, Kappelle LJ, van den Berg E, et al. Cognitive function in patients with diabetes mellitus: guidance for daily care. Lancet Neurol 2015;14:329-40.

9. Cheng G, Huang C, Deng H, et al. Diabetes as a risk factor for dementia and mild cognitive impairment: a meta-analysis of longitudinal studies. Intern Med J 2012;42:484-91.

10. Jones A, Kulozik P, Ostertag A, et al. Common pathological processes and transcriptional pathways in Alzheimer's disease and type 2 diabetes. J Alzheimers Dis 2009;16:787-808.

11. Swardfager W, Lanctôt K, Rothenburg L, et al. A metaanalysis of cytokines in Alzheimer's disease. Biol Psychiatry 2010;68:930-41.

12. Psilopanagioti A, Papadaki H, Kranioti EF, et al. Expression of adiponectin and adiponectin receptors in human pituitary gland and brain. Neuroendocrinology 2009;89:38-47.

13. Schrijvers EM, Witteman JC, Sijbrands EJ, et al. Insulin metabolism and the risk of Alzheimer disease: the Rotterdam Study. Neurology 2010;75:1982-7.

14. Gorska-Ciebiada M, Saryusz-Wolska M, Borkowska A, et al. Adiponectin, leptin and IL-1 $\beta$ in elderly diabetic patients with mild cognitive impairment. Metab Brain Dis 2016;31:257-66.

15. Diniz BS, Teixeira AL, Campos AC, et al. Reduced serum levels of adiponectin in elderly patients with major depression. J Psychiatr Res 2012;46:1081-5.

16. Song J, Lee JE. Adiponectin as a new paradigm for approaching Alzheimer's disease. Anat Cell Biol 2013;46:229-34.

17. Gorska-Ciebiada M, Saryusz-Wolska M, Borkowska A, et al. Serum levels of inflammatory markers in depressed elderly patients with diabetes and mild cognitive impairment. PLoS One 2015;10:e0120433. 
18. Marioni RE, Strachan MW, Reynolds RM, et al. Association between raised inflammatory markers and cognitive decline in elderly people with type 2 diabetes: the Edinburgh Type 2 Diabetes Study. Diabetes

Cite this article as: Liu H, Ma J, Sun L, Zhang Q, Fan J. Relationship between cognitive impairment and serum amyloid $\beta$-protein, adiponectin, and C-reactive protein levels in type II diabetes patients. Ann Palliat Med 2021;10(6):6502-6509. doi: 10.21037/apm-21-1074
2010;59:710-3.

(English Language Editor: L. Huleatt) 